\title{
The current landscape of television and movies in medical education
}

\author{
Marcus Law · Wilson Kwong · Farah Friesen · \\ Paula Veinot $\cdot$ Stella $\mathrm{L}$. Ng
}

Published online: 17 September 2015

(C) The Author(s) 2015. This article is published with open access at Springerlink.com

\begin{abstract}
Background Using commercially available television and movies is a potentially effective tool to foster humanistic, compassionate and person-centred orientations in medical students.
\end{abstract}

Electronic supplementary material The online version of this article (doi: 10.1007/s40037-015-0205-9) contains supplementary material, which is available to authorized users.

\footnotetext{
M. Law $(\bowtie)$

Department of Family \& Community Medicine, Faculty of Medicine, University of Toronto,

Room 2325, Medical Sciences Building, 1 King's College Circle, M5S 1A8 Toronto, ON, Canada

e-mail: marcus.law@utoronto.ca

S. L. Ng $\cdot$ F. Friesen $\cdot$ M. Law

Centre for Faculty Development,

Li Ka Shing Knowledge Institute, St. Michael's Hospital,

Toronto, ON, Canada

M. Law

Toronto East General Hospital,

Toronto, ON, Canada

W. Kwong · P. Veinot

Queen's University,

Kingston, ON, Canada

\section{S. L. Ng}

Department of Speech-Language Pathology,

University of Toronto,

Toronto, Ontario, Canada

\section{S. L. Ng}

Centre for Ambulatory Care Education,

Women's College Hospital,

Toronto, Ontario, Canada
}

Aim We reviewed pedagogical applications of television and movies in medical education to explore whether and why this innovation holds promise.

Methods We performed a literature review to provide a narrative summary on this topic.

Results Further studies are needed with richer descriptions of innovations and more rigorous research designs.

Conclusion As we move toward evidence-informed education, we need an evidence- based examination of this topic that will move it beyond a 'show and tell' discussion toward meaningful implementation and evaluation. Further exploration regarding the theoretical basis for using television and movies in medical education will help substantiate continued efforts to use these media as teaching tools.

Keywords Medical education - Movies - Television · Review

\section{Essentials}

- It is challenging to foster humanistic, compassionate and person-centred orientations in medical care and education.

- A potentially effective approach to this goal is the use of television and movies as educational tools.

- There is promise for television and movies to play a unique role in medical education in relation to fostering compassionate, critically conscious care orientations.

- Further exploration regarding the theoretical basis for using television and movies and objective evaluation of whether or not this intervention increases knowledge are suggested as avenues for future research. 


\section{Introduction}

Innovation in medical education continues to transform the way medical students learn in the classroom. While lecturebased teaching continues to be a staple of medical education, a variety of audio-visual media have also been introduced in the classroom setting. For example, some teachers use videos of actual or simulated events, scripted scenes for teaching purposes, and still images to enhance and support their teaching. There is evidence suggesting that, as early as the 1890 s, physicians recorded patients on film for the benefit of teaching medical students [1]. The appeal of using audio-visual media is apparent, even if its effectiveness is still to be determined. Further, emotions play a specific role in learning attitudes and behaviours, and audio-visual media such as television and movies can enhance emotions, making learning more memorable and pleasurable $[2,3]$. One might also speculate that a video format creates a degree of realism that is more conducive to learning about the humanistic factors in medicine $[4,5]$. This is particularly true of the specific audio-visual medium of television and movies, which has been an ongoing trend in medical education over the past two decades, in addition to the influx and rising popularity of dramatized medicine in popular culture $[6,7]$.

A number of articles have examined the use of either television or movie clips to teach medical students about a variety of different topics. The use of television and movies in medical education may be related to, and perhaps capitalizes upon, the highly emotional context in which medical education takes place. Medical education research has shown that students are more likely to learn and remember something when the teaching point is associated with an emotional response $[3,8]$. Outside of medicine, there is also a great deal of evidence that being in an emotional state can influence one's learning and memory pattern [9].

It is challenging to foster humanistic, compassionate and person-centred orientations in medical care and education [10], and we propose that a potentially effective approach to this goal is the use of television and movies as educational tools $[2,3]$. Although we hypothesize that emotions play a role in explaining why television and movies serve as an effective teaching tool, the pedagogical characteristics of this teaching format have not been formally evaluated in the academic literature. In line with efforts to ensure that innovation in medical education is evidence-informed [11], we sought to conduct a literature review on pedagogical applications of television and movies in medical education to explore why this innovation is so captivating for medical trainees. We examined the available evidence regarding why and how television and movies are used as a pedagogical modality, the various topics being taught, and the student outcomes and evaluations. Our goal was to better understand the utility of this modality and, in doing so, identify both its strength and weaknesses as a teaching tool, given that educators are likely to continue its use in medical education.

\section{Methods}

To critically evaluate the current landscape of the use of television and movies in medical education, we performed a literature review to provide a narrative summary on this topic [12].

\section{Search strategy}

We performed searches in PubMed and OVID Medline databases from inception to 21 October 2014, using a combination of MeSH (education, medical; students, medical; faculty, medical; motion pictures as topic; television) and keyword searches (medical education; movie*; motion picture; drama; $\mathrm{TV}$; television; cinemeducation). We also reviewed the tables of content of select journals from January 2000 to October 2014, including early online publications (Medical Teacher, Medical Education, Teaching and Learning in Medicine, Advances in Medical Education and Practice and Academic Medicine). We footnote chased the reference lists of articles included for full-text review, which were imported into a citation management system (EndNote) with any duplicates removed.

\section{Article selection}

Two authors (WK, FF) identified relevant articles for fulltext review by examining the titles and abstracts of the search results. We included research, review and opinion articles in English. Articles were included if they discussed the use of commercially available television and movies (including documentaries) in medical education. Articles related to non-medical health professions education and the influence of television and movies on public perception were excluded. Articles discussing medical students' television or movie viewing habits, or why these media are interesting were excluded, as the main focus of the article is how these media are used to educate medical students. Articles which only briefly mentioned television or movies in the context of a general discussion on humanities in health care were also excluded (Fig. 1). Each article included for full-text review was independently reviewed with data abstracted by two authors (WK, FF). Data abstraction fields included authors, title, journal, year of publication, intervention (television or movies), purpose of intervention, study design, participants, outcomes, key findings, and Kirkpatrick's four levels of change model [13]. 
Fig. 1 Search Strategy Flow Chart

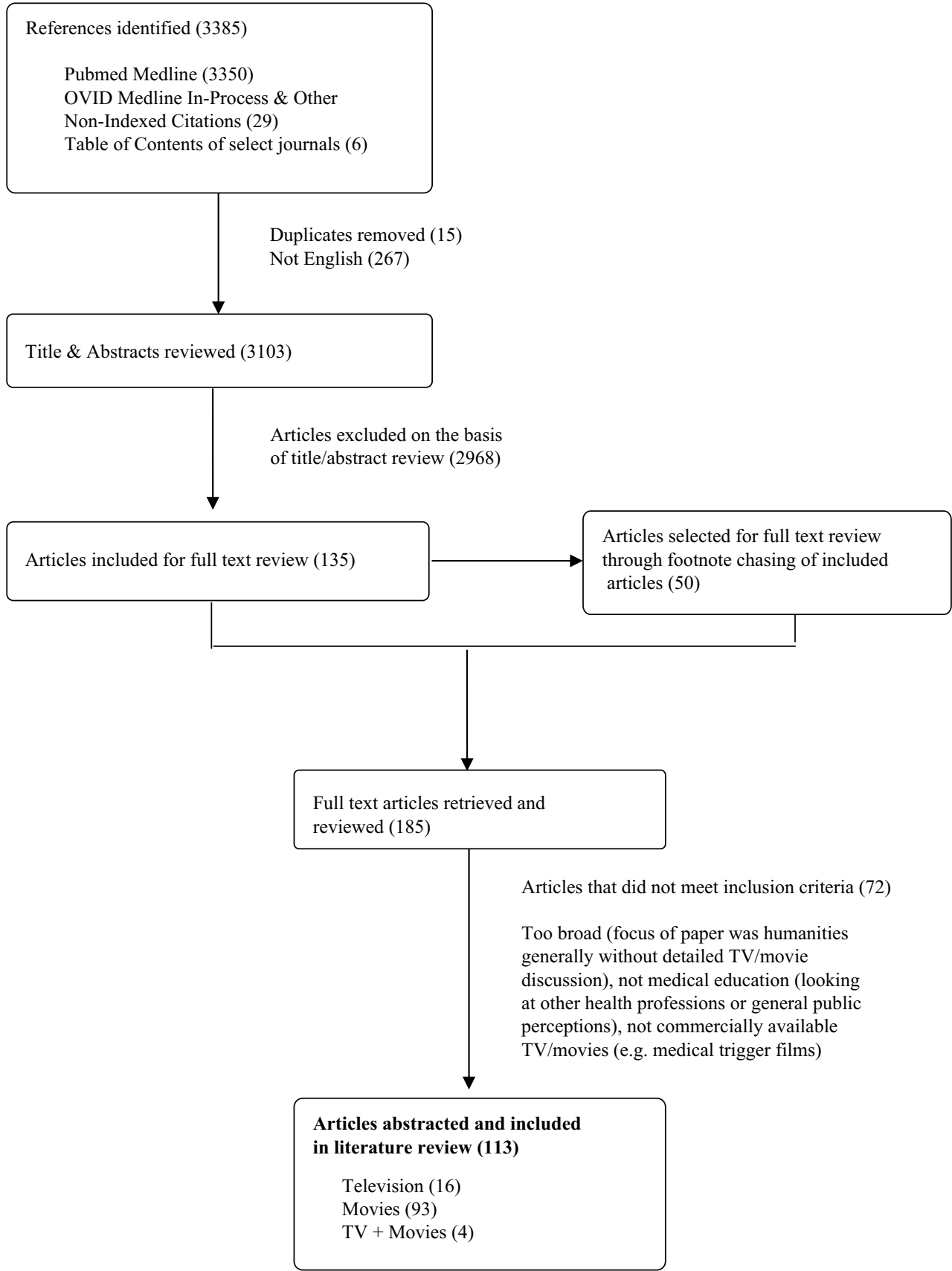

Purpose (Why?)

The papers reviewed described television and movies being used in medical education to convey factual information to attain knowledge, facilitate skills acquisition, enrich learning, maintain interest and engagement, encourage debate and discussion, motivate reflection, evoke emotion and stimulate memory retention. These purposes were similar for both media. purpose did they serve? What content was presented? In what formats were they used? What were the outcomes or evaluation results? 
Topics/Content (What?)

With respect to television, the majority of academic work investigating medicine in art and entertainment stems from medical dramas. The idea of utilizing television medical dramas as a novel teaching tool has been postulated and discussed through many lenses, but few studies have actually tested the idea in practice. The following were evaluated in course content using television shows: teaching students how to break bad news and deal with difficult situations [14]; and professionalism, including teamwork and communication skills $[15,16]$. Ethics was a main topic of teaching for many studies $[6,7,17]$, with a focus on psychiatry/ mental health in several studies, including teaching psychotherapy [14, 18]. Empathy was the topic of many studies [19-23]. Doctor-patient communication or interactions was also examined [21, 24].

In the papers reviewed regarding the use of movies in medical education, psychiatry/mental health [25-32] dominated as educational topics. Hollywood movies, documentaries, and short films were screened in full or shown as clips to teach students on topics such as professionalism [21, 22, 33], ethics [34-36], cultural competence [5, 37, 38], managing severe or terminal disease $[23,24]$, breaking bad news [14], death and dying [24], and alcohol and other drugs [39, 40]. The subjects of sexuality [41] and clinical pharmacology [42] were raised in select studies. Specific disease topics mentioned were cancer [24], epilepsy [29], and AIDS [23]. For details about each study,

\section{Pedagogy/Format (How?)}

The articles described a range of pedagogical approaches. While interventions and curricular designs were generally described with limited detail, most articles reported using television and movies to spark discussion. Students either watched television shows and movies in full or viewed a relevant clip. In some cases, the media presentation was followed by a lecture/seminar on the topic and a subsequent discussion in a large group format. Many studies reported on students' forming small groups for problem-based or case-based learning. In a few instances, students completed reflective essays or narratives. In some, students were asked to conduct presentations or role-play. A question and answer session with the producers of the film, a multiple-choice format to stimulate discussion and recordings of therapy sessions were each used in single studies. Many studies reported their findings as independent, stand-alone educational events.
Outcomes and evaluation

There were three main types of articles: (1) commentary/ perspective encompassing editorials, letters, and general summaries (guides) for television or movies to use for specific topics, which can be quite detailed, as in Hyler and Schanzer's (1997) guide to 33 movies that depict borderline personality disorder [28]; (2) course descriptions, which describe courses/seminars/workshops, but may not provide specific details about implementation or participants and in which formal evaluation (if any) is not reported; and (3) research or evaluation with clear study design description and outcomes (e.g., literature or systematic review, randomized controlled trials, pre-and post- or pre/post surveys, questionnaires, or tests).

Across our dataset, there were 35 perspective, 43 description, and 35 research/evaluation articles and, overall, most articles were in support of using television and movies in medical education. The studies reviewed suggest television and media use in medical education serves a number of purposes, namely knowledge attainment, skills acquisition, learning enrichment, interest and engagement, debate and discussion, reflection, emotion provocation, and memory retention. The research or evaluation studies examined short-term outcomes and tended to measure student satisfaction and knowledge and, to a lesser extent, attitudes (or some combination). Studies used evaluation methods consistent with Kirkpatrick levels 1 'reaction' (satisfaction) and 2 'learning' (change in attitudes, knowledge).

All studies measuring satisfaction indicated that students were satisfied with the use of television and movies for the purposes of medical education. When knowledge on a subject was evaluated before and after viewing clips, students subjectively reported that the use of entertainment added to their knowledge base. Of the 113 articles abstracted, only three argued against using entertainment media as a teaching tool in medical education [43-45]. The remaining 110 articles reiterated findings from previous studies and commented on the potential to use media clips in the classroom. Those articles not in favour of this teaching methodology expressed concern that ethical encounters and mental health patients were not accurately portrayed in television shows and, therefore, the use of these media to generate discussion on the subject was inherently flawed [43-45].

\section{Discussion}

The use of various media in teaching to address a range of outcomes is not new. In a paper on how video clips embedded in multimedia presentations (including television and movies) can be used to improve learning in college courses, Berk (2009) proposed that the use of clips can attain 20 spe- 
cific learning outcomes, such as gaining students' attention, focusing concentration, fostering creativity, stimulating the flow of ideas, making learning fun and decreasing anxiety and tension on upsetting topics, among others [46]. By employing video clips that tap into multiple intelligences and a 'pluralistic view of the mind', faculty expose students to a range of learning strategies utilizing verbal, visual and auditory stimuli [46].

In developing their medical expertise and intrinsic competencies (i.e., communication, collaboration, professionalism), medical students are required to master a range of topics. The papers we reviewed highlight an array of subjects covered by this teaching modality, suggesting broad application of this instructional tool across topic areas. Empathy, the focus of many studies [19-23], was the most frequently examined component underlying a person-centred and humanistic approach to medicine. This suggests the importance of this skill and would certainly play a role in doctor-patient communication or interactions, a similarly studied topic [21, 24].

This review found that a range of pedagogical techniques are applied, including watching television shows and movies in full or a relevant clip, accompanied by a large group discussion, small group sessions for problem-based or casebased learning, reflective essays or narratives, presentations or role-play. The varied use of television and movies in medical education may imply that no single pedagogical technique will achieve $100 \%$ effectiveness across all learning styles and all competencies a student is expected to attain. Therefore, television and movies need to be employed with careful consideration of the learning objectives expected to be achieved and the intended outcomes, lest they take time away from better proven educational approaches.

There are simultaneous calls for evidence-informed education and a greater emphasis on a person-centred, humanistic approach to medicine. Therefore, building on the studies we reviewed, there is a need to study approaches that foster competencies associated with the intrinsic roles of humanistic clinicians. Some have advocated efforts to help learners move beyond rote or surface performance of desirable 'knowledge, skills, and attitudes' toward a genuine critical consciousness, an embodiment of virtues, and an ability to recognize power differentials, and patients, as individuals influenced by complex life histories and social structures $[4,47]$. Teaching related to these topics may benefit from thoughtful applications of pedagogical innovations such as television and movies.

Findings suggest that utilizing television and movies to evoke emotions in trainees stimulates their own emotional awareness thus allowing them to be more empathic with patients. Television and movies are said to heighten learners' understanding of situational ethics and human suffering [34] and to help them to reflect upon the humanitarian (emotional and psychological) aspects of medicine [48]. As an effective teaching tool, television and movies seem to evoke emotions that stimulate learning about the moral and emotional issues that are part of practising medicine [49]. By appealing to the affective domain of learning, this pedagogical tool may serve to develop trainees' awareness of their own emotions and empathy, subsequently permitting a better understanding of how to offer emotional support to patients.

A few areas of improvement are needed in future studies assessing the effectiveness of television and movies in medical education. Aside from utilizing post-session evaluation, no study has compared the use of entertainment in the classroom to the standard pedagogical method (i.e., lectures, small groups). Assessing whether or not students actually prefer the use of entertainment to traditional teaching methods, or whether such practices result in an improvement in course performance would be worthwhile.

When implementing such methods, it is important to consider long-term evaluation that goes beyond measuring satisfaction, attitudes and self-perceived knowledge to address Kirkpatrick's levels 3 (behaviour) and 4 (results). This means measuring how knowledge and skill acquisition translates into behaviour change (clinical application) and thus improved results (patient care). For example, do medical students behave more empathically towards patients?

From a research and evaluation point of view, further studies are needed with richer descriptions of innovations and more rigorous research designs. Additionally, these studies need to fully describe how educational events are incorporated or integrated into the full curriculum. Inadequate detail of such innovations and their evaluation makes it difficult to truly understand what has taken place, and thus to make an informed judgment about their value [50]. Ensuring that an intervention is fully and unambiguously described will encourage curriculum developers to engage in evidence-based curriculum design and employ a sound theoretical framework. Using television and movies in medical education, like eLearning, should not be done solely for novelty. For such innovations to be effective, there needs to be an identified learning gap, a defined theory and a curriculum design that utilizes technology, if appropriate, to address the gap.

Furthermore, given its powerful cognitive and emotional impact, media is a potentially valid approach to tap into the multiple intelligences and learning styles of the 'net generation' [46]. The caveat is that, as technology becomes ever more pervasive in society, and as use of emerging media tools (e.g., social media) becomes increasingly widespread, there is greater pressure on faculty to integrate media into their teaching. With such emphasis, careful attention is required to ensure that faculty are adequately prepared to 
use this instructional tool to deliver quality educational opportunities to medical students.

\section{Conclusion}

There is promise for television and movies to play a unique role in medical education in relation to fostering compassionate, critically conscious care orientations. Some evidence suggests that students find this form of teaching engaging, but further work is needed to justify and facilitate the implementation of this teaching format into curricula, including adequate preparation of faculty. Because a visual form of entertainment is readily available and wellinscribed into popular culture [7], the student population can easily accept and enjoy the use of television and movies throughout their medical education. But if we are to strive toward evidence-informed education, we need an evidence base on this topic to move beyond 'show and tell' toward meaningful implementation and evaluation. Further exploration regarding the theoretical basis for using television and movies in medical education will help substantiate continued efforts to use these media as teaching tools.

\section{Declaration of Interest The authors report no declarations} of interest and no sources of support.

Open Access This article is distributed under the terms of the Creative Commons Attribution License which permits any use, distribution, and reproduction in any medium, provided the original author(s) and source are credited.

\section{References}

1. Essex-Lopresti M. Centenary of the medical film. Lancet. 1997;349:819-20.

2. Blasco PG, Moreto G, Roncoletta AFT, Levites MR, Janaudis MA. Using movie clips to foster learners' reflection: improving education in the affective domain. Fam Med. 2006;38:94-6.

3. Taylor JS. Learning with emotion: a powerful and effective pedagogical technique. Acad Med. 2010;85:1110.

4. Kumagai AK. From competencies to human interests: ways of knowing and understanding in medical education. Acad Med. 2014;89:978-83.

5. Ross PT, Kumagai AK, Joiner TA, Lypson ML. Using film in multicultural and social justice faculty development: scenes from Crash. J Contin Educ Health Prof. 2011;31:188-95.

6. Hirt C, Wong K, Erichsen S, White JS. Medical dramas on television: a brief guide for educators. Med Teach. 2013;35:237-42.

7. Czarny MJ, Faden RR, Nolan MT, Bodensiek E, Sugarman J. Medical and nursing students' television viewing habits: potential implications for bioethics. Am J Bioeth. 2008;8:1-8.

8. McConnell MM, Eva KW. The role of emotion in the learning and transfer of clinical skills and knowledge. Acad Med. 2012;87:1316-22.

9. Heuer F, Reisberg D. Vivid memories of emotional events: the accuracy of remembered minutiae. Mem Cognit. 1990;18:496-506.
10. Cohen LG, Sherif YA. Twelve tips on teaching and learning humanism in medical education. Med Teach. 2014;36:680-4.

11. Van der Vleuten CPM, Driessen EW. What would happen to education if we take education evidence seriously? Perspect Med Educ. 2014;3:222-32.

12. Grant MJ, Booth A. A typology of reviews: an analysis of 14 review types and associated methodologies. Health Info Libr J. 2009;26:91-108.

13. Kirkpatrick D. Great ideas revisited. Train Dev. 1996;50:54-9.

14. Mcneilly DP, Wengel SP. The 'ER' seminar: teaching psychotherapeutic techniques to medical students. Acad Psychiatry 2001;25:193-200.

15. Wong RY, Saber SS, Ma I, Roberts JM. Using television shows to teach communication skills in internal medicine residency. BMC Med Educ. 2009;9:9.

16. Shevell AH, Thomas A, Fuks A. Teaching professionalism to first year medical students using video clips. Med Teach. 2014;1-8.

17. Arawi T. Using medical drama to teach biomedical ethics to medical students. Med Teach. 2010;32:e205-10.

18. Aboul-Fotouh F, Asghar AA. Therapy 101: a psychotherapy curriculum for medical students. Acad Psychiatry. 2010;34:248-52.

19. Hojat M, Axelrod D, Spandorfer J, Mangione S. Enhancing and sustaining empathy in medical students. Med Teach. 2013;35:996-1001.

20. Lenahan P, Shapiro J. Facilitating the emotional education of medical students: using literature and film in training about intimate partner violence. Fam Med. 2005;37:543-5.

21. Klemenc-Ketis Z, Kersnik J. Using movies to teach professionalism to medical students. BMC Med Educ. 2011;11:60.

22. Gramaglia C, Jona A, Imperatori F, Torre E, Zeppegno P. Cinema in the training of psychiatry residents: focus on helping relationships. BMC Med Educ. 2013;13:90.

23. Goldman JD. An elective seminar to teach first-year students the social and medical aspects of AIDS. J Med Educ. 1987;62:557-61.

24. Ozcakir A, Bilgel N. Educating medical students about the personal meaning of terminal illness using the film, 'Wit.' J Palliat Med. 2014;17:913-7.

25. Akram A, O'Brien A, O'Neill A, Latham R. Crossing the line-learning psychiatry at the movies. Int Rev Psychiatry. 2009;21:267-8.

26. Bhagar HA. Should cinema be used for medical student education in psychiatry? Med Educ. 2005;39:972-3.

27. Fritz GK, Poe RO. The role of a cinema seminar in psychiatric education. Am J Psychiatry. 1979;136:207-10.

28. Hyler SE, Schanzer B. Using commercially available films to teach about borderline personality disorder. Bull Menninger Clin. 1997;61:458-68.

29. Kalra G. Teaching diagnostic approach to a patient through cinema. Epilepsy Behav. 2011;22:571-3.

30. Kalra G. Psychiatry movie club: a novel way to teach psychiatry. Indian J Psychiatry. 2011;53:258.

31. Rieder RO. Teaching about schizophrenia. Schizophr Bull. 1974;1(9):5-9.

32. Tarsitani L, Brugnoli R, Pancheri P. Cinematic clinical psychiatric cases in graduate medical education. Med Educ. 2004;38:1187.

33. Lumlertgul N, Kijpaisalratana N, Pityaratstian N, Wangsaturaka D. Cinemeducation: a pilot student project using movies to help students learn medical professionalism. Med Teach. 2009;31:e327-32.

34. Quadrelli S, Colt HG, Semeniuk G. Appreciation of the aesthetic: a new dimension for a medicine and movies program. Fam Med. 2009;41:316-8.

35. Self D, Baldwin D, Olivarez M. Teaching medical ethics to firstyear students by using film discussion to develop their moral reasoning. Acad Med. 1993;68:383-5. 
36. Volandes A. Medical ethics on film: towards a reconstruction of the teaching of healthcare professionals. J Med Ethics. 2007;33:678-80.

37. Murphy-Shigematsu S, Grainger-Monsen M. The impact of film in teaching cultural medicine. Fam Med. 2010;42:170-2.

38. Sierles FS. Using film as the basis of an American culture course for first-year psychiatry residents. Acad Psychiatry. 2005;29:100-4.

39. Welsh CJ. OD's and DT's: using movies to teach intoxication and withdrawal syndromes to medical students. Acad Psychiatry. 2003;27:182-6.

40. Pais de Lacerda A. Medical education: addiction and the cinema (drugs and gambling as a search for happiness). J Med Movies. 2005; 1:95-102.

41. Mandel JB. Medical education about human sexuality: the impact of film in the workshop setting. Med Educ. 1983;17:305-10.

42. Koren G. Awakenings: using a popular movie to teach clinical pharmacology. Clin Pharmacol Ther. 1993;53:3-5.

43. Trachtman H. The medium is not the message. Am J Bioeth. 2008;8:9-11.

44. Greenberg HR. Caveat actor, Caveat emptor: some notes on some hazards of Tinseltown teaching. Int Rev Psychiatry. 2009;21:241-4.

45. Ramchandani $\mathrm{D}$. The downside of teaching psychopathology with film. Acad Psychiatry. 2012;36:154-5.

46. Berk RA. Multimedia teaching with video clips: TV, movies, YouTube, and mtvU in the college classroom. Int J Technol Teach Learn. 2009;5:1-21.
47. Kumagai AK, Lypson M. Beyond cultural competence: critical consciousness, social justice, and multicultural education. Acad Med. 2009;84:782-7.

48. Loscos J, Baños J, Loscos F, la Cámara J de. Medicine, cinema and literature: a teaching experiment at the Universitat Autonoma de Barcelona. J Med Movies. 2006;2:138-42.

49. Blasco PG, Mônaco CF, De Benedetto MAC, Moreto G, Levites MR. Teaching through movies in a multicultural scenario: overcoming cultural barriers through emotions and reflection. Fam Med. 2010;42:22-4.

50. Ellaway R. Apples and architraves: a descriptive framework for e-learning research. Med Teach. 2010;32:95-7.

Marcus Law, MD, MBA, MEd is Director of Preclerkship Renewal, Undergraduate Medical Education, University of Toronto.

Wilson Kwong, MSc is a final year medical student, Queen's University.

Farah Friesen, MI is research coordinator, Centre for Faculty Development, University of Toronto.

Paula Veinot, MHSc is an independent research consultant.

Stella L. Ng, PhD is Director of Research, Centre for Faculty Development, University of Toronto. 